\title{
Aplicación de los indicadores de calidad y contaminación del agua en la determinación de la oferta hídrica neta
}

ENVIRONMENTAL ENGINEERING

\section{Application of the quality indicators and contamination of the water in the determination of net hydric supply}

\author{
Natalia E. Samboni", Aldemar Reyes T. ${ }^{\S^{*}}$, Yesid Carvajal E* \\ *Escuela de Ingeniería de Recursos Naturales y del Ambiente, Universidad del Valle, Cali, Colombia \\ saldemar.reyes@correounivalle.edu.co,irehisa@univalle.edu.co, \\ yesid.carvajal@correounivalle.edu.co
}

(Recibido: Abril 13 de 2010 - Aceptado: Septiembre 26 de 2011)

\begin{abstract}
Resumen
La oferta hídrica neta hace referencia al agua que emplea el hombre para sus diferentes usos como: agricultura, industria, doméstico y recreación; en Colombia se determina aplicando la Resolución 0865 del 2004 del Ministerio de Ambiente, Vivienda y Desarrollo Territorial, donde a la oferta superficial se aplican dos factores de reducción correspondientes a un $25 \%$ por caudal ecológico y $25 \%$ por calidad del agua. La aplicación de estas dos reducciones se ha limitado en la mayoría de los casos, al aplicar estos porcentajes al caudal medio mensual multianual más bajo, por lo tanto, los estudios de calidad no se incluyen directamente en este cálculo. En este artículo se presenta la metodología para calcular la reducción correspondiente a la calidad del agua, a partir de la determinación de los indicadores de calidad y contaminación, ICA e ICO, utilizando información de dos subcuencas tributarias al río Cauca: Zanjón Oscuro y Río Hondo, localizadas en la cuenca alta del río Cauca, encontrando una relación directa entre la reducción y la contaminación del agua.
\end{abstract}

Palabras Clave: Oferta hídrica neta, Calidad del agua, Indicadores de calidad, Contaminación del agua.

\begin{abstract}
The net water supply refers to water employed by the man for various uses including agriculture, industry, domestic and recreation; in Colombia, is defined by Resolution 0865 of 2004 the Ministry of Environment, Housing and Territorial Development, where supply surface use two different reduction factors: $25 \%$ for environmental flow and $25 \%$ for water quality. The application of these two reductions is limited in most cases, applying these percentages to the lowest average monthly flow multiyear, therefore, quality studies are not directly included in this calculation. This paper presents the methodology for calculate the reduction for water quality, from the identification of appropriate quality and pollution, ICA, ICO, using information from two sub-basin tributaries of the Cauca river basin: Hondo river and Zanjón Oscuro, located in the upper Cauca River basin, finding a direct relationship between the reduction and water pollution.
\end{abstract}

Keywords: Net hydric supply, Water quality, Indicators of quality, Water contamination 


\section{Introducción}

El desarrollo de las civilizaciones ha estado marcado por un factor: la presencia o ausencia del agua. Si está presente y en abundancia, el agua representa la posibilidad de mejoramiento agrícola, social, industrial, sanitario y de la calidad de vida. Si el recurso hídrico está ausente o escasea, es motivo de pobreza, guerras, enfermedades y estancamiento económico (Sommer, 2000).

Los encargados de la formulación de políticas han concentrado su atención en los tres usos principales del agua: la industria, la agricultura y el uso doméstico; ignorando el cuarto usuario primordial del agua, el medio ambiente. Por esta razón hoy es claro que el manejo del agua ha dejado de ser un asunto exclusivamente técnico para dar paso a una visión más compleja e interdisciplinaria denominada "Gestión Integral del Recurso Hídrico - GIRH", que parte de la idea; para que el manejo del agua sea sostenible, debe incluir el ciclo hidrológico completo, proteger e incrementar la oferta natural y considerar en conjunto las demandas, la calidad y necesidades de todos sus usuarios (Guhl, 2006).

Colombia posee un potencial hídrico tres veces mayor que el promedio suramericano y seis veces mayor que la oferta hídrica específica promedio mundial (IDEAM, 2010), pero ha empezado a tener problemas de disponibilidad de agua en muchas de las regiones, especialmente en las más pobladas, debido a la contaminación, deforestación, la erosión, la pérdida de la capacidad de retención y regulación del recurso en los suelos de laderas y piedemonte, que han afectado la biodiversidad y los ecosistemas boscosos y de páramo, repercutiendo en un proceso lento de desestabilización hídrica en las grandes cuencas, tales como las de los ríos Magdalena y Cauca (Marín, 2003).

La cantidad de agua disponible para diferentes usos en una cuenca o unidad hidrológica se denomina "oferta hídrica neta", en Colombia la resolución 0865 del 2004 (MAVDT,2004) establece la "Metodología para la determinación del índice de escasez para aguas superficiales", donde se propone que a la oferta hídrica total se le deben aplicar dos factores de reducción: uno por calidad de agua del $25 \%$ y otro por caudal ecológico del $25 \%$, valores que conforman el llamado caudal ambiental, cálculos que se han limitado a solo aplicar dichos porcentajes al caudal medio mensual multianual más bajo y no relacionan directamente la calidad con la cantidad (Samboni, 2009).

Se considera entonces que el caudal que permanece en la corriente durante el $75 \%$ del tiempo representa el mínimo que podría fluir por el cauce una vez se realicen las captaciones. Sin embargo, en el Índice de Escasez, el régimen de caudal ambiental sigue siendo flexible, dando opción de disponer de un estudio técnico y fundamentado del tema, o de hacer cambios si éste afecta la viabilidad del proyecto (Castro, et al. 2006). La falta de reglamentación y conocimiento en este sentido permite al diseñador, conservar solo el 10\% del caudal medio anual del río y, en algunos casos, del mínimo anual; criterio que no tiene fundamento teórico, ni técnico y es una copia de reglamentaciones extranjeras (Castro \& Carvajal, 2009), donde en muchos casos no se considera que la distribución de la precipitación en Colombia está influenciada por la variabilidad espacio temporal de la Zona de Convergencia Intertropical, por los sistemas de circulación de la atmósfera y por el relieve, entre otros; los cuales generan la diversidad climática del país.

Tampoco considera el efecto del fenómeno ENOS sobre la hidroclimatología con fuertes impactos en las cuencas andinas y del Pacífico, ante el aumento de probabilidad de que se produzcan eventos extremos, (Magaña, 2004); estas características deben tomarse en cuenta en la aplicación de metodologías hidrológicas desarrolladas en otras latitudes, incluyendo además la variabilidad hidrológica, es decir que oscile intra e interanualmente, incluyendo caudales especiales como las crecidas periódicas, eventos extremos, caudales basales, etc, (Gottschalk \& Krasovskaia, 2010); tan importantes para las especies biológicas como para la vegetación ribereña, la formación del cauce, la descontaminación de las aguas y la configuración del espacio biofísico de muchas especies (Palau, 2003). 
La calidad del recurso hídrico puede ser determinada a partir de los índices de calidad (ICA), como el elaborado por "The National Sanitation Foundation" de los Estados Unidos "ICANSF" usado para la evaluación de fuentes de abastecimiento y los indicadores de contaminación del agua (ICO), como el elaborado en Colombia por Ramírez et al., (1998) "ICOMO", utilizado en la evaluación contaminación orgánica.

Los ICA e ICO, consisten básicamente en una expresión matemática simple, de la combinación de un número de parámetros físicos, químicos y/o microbiológicos, los cuales sirven como medida de la calidad del agua para diferentes usos (Fernández \& Solano, 2005). Finalmente el valor numérico obtenido, que oscilan entre 0 a 100 y 0 a 1 , se clasifica en diferentes rangos a los cuales se le asigna una descripción cualitativa del grado de contaminación del agua, con los cuales puede valorarse el recurso (Samboni, et al, 2007).

El objetivo de este trabajo es proponer una metodología que incluya la evaluación de calidad del agua en la determinación de la oferta hídrica neta, donde los rangos de calidad obtenidos a partir de los indicadores ICA e ICO son relacionados directamente con la reducción por calidad, así: la calidad es mala, cuando la reducción es cercana al $100 \%$, esto indica que el agua es muy contaminada y por lo tanto no hay disponibilidad del recurso para uso agrícola, industrial, doméstico y recreación; en el caso de una baja contaminación la reducción asignada es cercana al $0 \%$ indicando que puede ser empleada en los principales usos.

\section{1 Índice de escasez del agua}

Mediante la resolución 0865 del 2004 emitida por el Ministerio de ambiente, Vivienda y Desarrollo Territorial, por la cual se adopta la metodología para el cálculo del índice de escasez para agua superficial, se define el índice de escasez mediante la Ec(1):

$$
\text { Ies }=\frac{\mathrm{Dh}}{\mathrm{Oh}} \times \mathrm{Frx100}
$$

Donde, Ies: Índice de escasez en porcentaje; Dh: Demanda hídrica en metros cúbicos $\left(\mathrm{m}^{3}\right)$; Oh: Oferta hídrica superficial neta en metros cúbicos $\left(\mathrm{m}^{3}\right)$; Fr: Factor de reducción por calidad del agua y el caudal ecológico.

Para estimar este índice resulta natural utilizar la anterior relación porcentual entre la demanda de agua del conjunto de actividades socioeconómicas y la oferta hídrica disponible en las fuentes abastecedoras. Esta relación es usualmente denominada Índice de Escasez y en los casos en que la demanda de agua representa más del $20 \%$ de la oferta de agua disponible en una región, permite activar las señales necesarias para implementar las acciones de gestión del recurso hídrico que aporten al desarrollo sostenible en el área de influencia (Rivera, et al. 2004; OEA, 2004).

Dado lo anterior, no solamente se debe tener en cuenta la relación entre la oferta y demanda de agua en una unidad de análisis, sino que es necesario tener presente que como mínimo en la fuente debe quedar un remante de agua capaz de garantizar las características de los caudales mínimos históricos y de abastecer la protección de las fuentes frágiles o vulnerables. Bajo este contexto, el índice de escasez refleja la relación de oferta y demanda de agua incluyendo las reducciones necesarias para mantener la salud de la fuente abastecedora (CRC \& UNIVALLE, 2007).

\subsection{Calidad del agua y escasez}

Durante el último siglo la población mundial se ha triplicado, mientras que el consumo de agua se ha sextuplicado. Aunque el tema de la escasez del agua, su contaminación y su distribución desigual es un problema de todos, afecta más a la población pobre del mundo. Hoy, los niños de los países desarrollados consumen entre 20 y 30 veces más cantidad de agua que los niños de los países más pobres. Se calcula que unos mil millones de personas no disponen de agua potable, en tanto dos mil quinientos millones no la tienen en las cantidades y condiciones mínimas adecuadas. Casi todas ellas viven en América Latina, África y Asia, aunque también existen vastos sectores de menesterosos en los países más desarrollados a quienes alcanza esta carencia (Yepe, 2008). 
A nivel mundial, el caso de Latinoamérica es diferente en cuanto a la cantidad, debido principalmente a la precipitación, lo que produce un gran excedente hídrico. El problema es de distribución, por ejemplo: Perú es un país que está situado en el parámetro de "suficiente", su disponibilidad per-cápita de agua hoy es de aproximadamente $1,790 \mathrm{~m}^{3} /$ año, sin embargo, la proyección es que en el año 2025 su disponibilidad será de $980 \mathrm{~m}^{3} /$ año, lo que implica una situación de stress. Países como Brasil, Bolivia, Colombia, Venezuela, Argentina y Chile se sitúan en el parámetro de los países "ricos", esto es, tienen entre 10,000 y $100,000 \mathrm{~m}^{3} /$ persona/año. La Guyana Francesa se sitúa en la franja de los "muy ricos", esto es, sobre $100,000 \mathrm{~m}^{3} /$ persona/año (Malvezzi, 2006).

El problema principal del agua en Colombia es, por tanto, un asunto de calidad antes que de cantidad y la principal contaminación proviene de los patógenos y nutrientes generados por la población urbana y el sector agrícola, antes que de los vertimientos industriales (Guhl, 2006). Según el Estudio Nacional del Agua, realizado por el Instituto de Hidrología, Meteorología y Estudios Ambientales (IDEAM), en caso de continuar la misma situación de presión a las fuentes de agua, para el año 2015 el 66\% de los colombianos podría estar en alto riesgo de sufrir escasez de agua (IDEAM, 2005).

\section{Metodología}

\subsection{Selección zona de estudio}

La Subcuenca Zanjón Oscuro está ubicada al norte del departamento del Cauca, tienen un área de $95.59 \mathrm{~km}^{2}$, una elevación media de 976 metros sobre el nivel del mar (msnm) y una pendiente media de $0.04 \mathrm{~km}^{2} / \mathrm{km}^{2}$ (CRC \& UNIVALLE, 2007). Se destaca por que en ella se encuentran localizados algunos de los principales ingenios del país. Se forma de la derivación tres ríos: del río Desbaratado, que en su margen izquierda permite el nacimiento a La Acequia Santa Ana, quien a su vez se une con Pitayo para formar el Zanjón Oscuro. El principal centro urbano que se localiza sobre esta subcuenca es el municipio de Puerto Tejada con una población de 44,220 habitantes según Censo (DANE, 2005), y asentamientos pequeños inferiores a los 500 habitantes como Santa Ana, Tulipán, Zanjón Oscuro, El Ortigal y las veredas Rico, Méjico y Hormiguero. El uso del agua es netamente agrícola y recibe las descargas industriales y domésticas de Santa Ana, el Ortigal y del $80 \%$ de la población de Puerto Tejada (CRC \& FONAN, 2000; CRC, 2001).

La Subcuenca Río Hondo tiene influencia en los municipios de Sotará, Timbío, Popayán y El Tambo, tienen un área de $343.27 \mathrm{~km}^{2}$, una elevación media de 1966 msnm y una pendiente media de $0.22 \mathrm{~km}^{2} / \mathrm{km}^{2}$ (CRC \& UNIVALLE, 2007). Se encuentra ubicado en una de las zonas hídricamente más ricas de la cuenca Alto Cauca. Río Hondo está formado por la confluencia de los ríos Negro y Sucio en el sector conocido como Los Dos Brazos, sector rural del municipio de Popayán, sus principales tributarios son los ríos Robles y Piedras. En la parte alta de la subcuenca se ubican varias bocatomas de acueductos veredales y distritos de riego que surten a los municipios aledaños; en la zona media y baja se usa para extracción de materiales y recreación. Recibe aguas residuales de las veredas Torres, La Playa y Figueroa que no tienen una red de alcantarillado (Samboni, 2009).

En las Figuras 1 y 2 se observa la ubicación de cada una de las subcuencas y la localización de los puntos de monitoreo.

A partir del comportamiento de la precipitación media mensual anual se determinó la distribución de la temporalidad en cada una de las subcuencas (Figura 3), encontrando un régimen de precipitaciones de dos máximos al año entre los meses de marzo a mayo y octubre a noviembre, siendo esta última la más intensa con promedios de $208 \mathrm{~mm}$ para Zanjón Oscuro y $316 \mathrm{~mm}$ para Río Hondo; y dos periodos de baja precipitación entre los meses de junio a septiembre y de diciembre a febrero, siendo el mes de julio el más bajo con promedio de $43 \mathrm{~mm}$ en Zanjón Oscuro y 57 mm en Río Hondo.

Teniendo en cuenta el régimen de precipitaciones se determinó el periodo de muestreo sobre la corriente principal en cada una de las subcuencas así: 


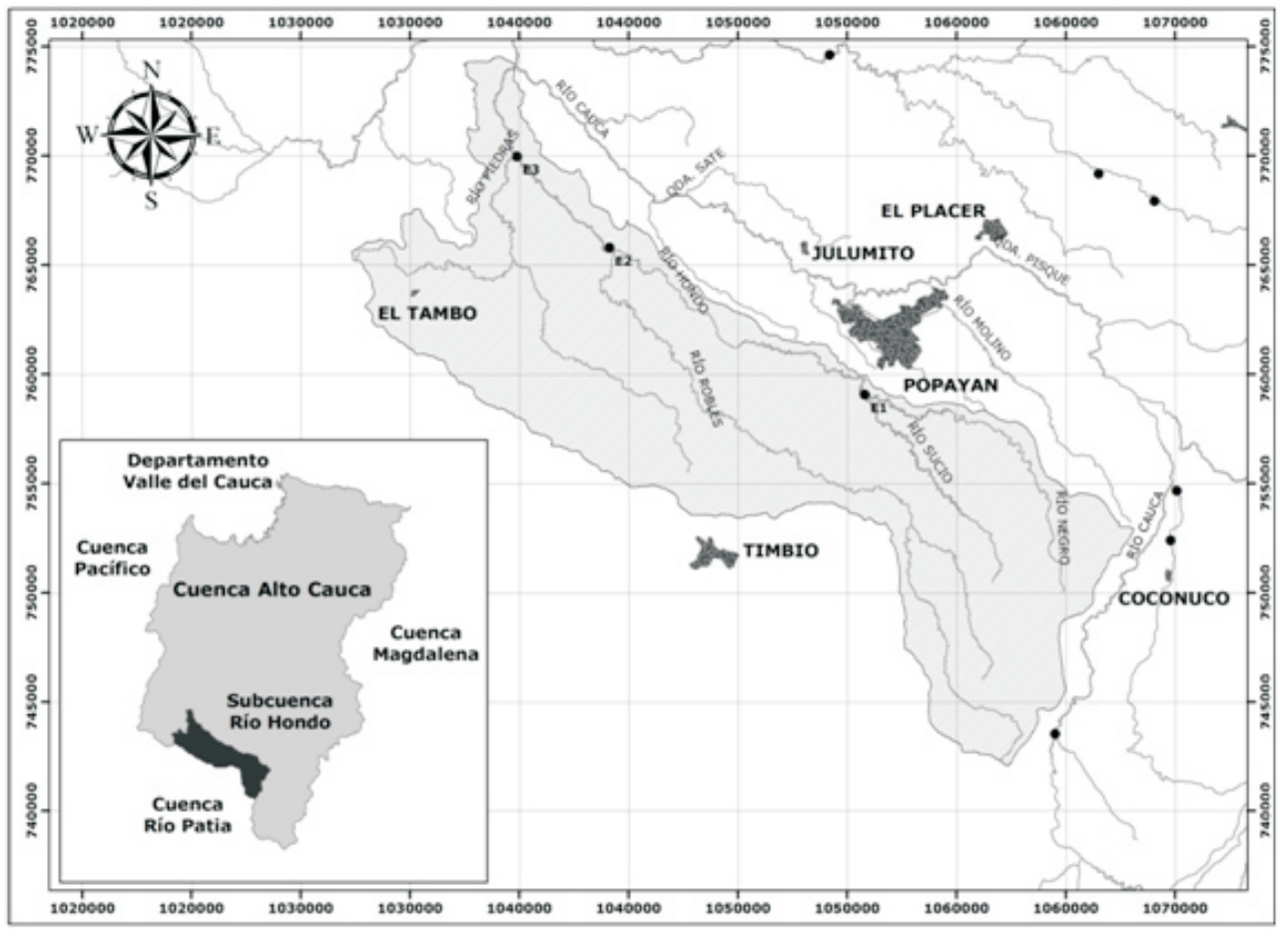

Figura 1. Localización Subcuenca Río Hondo

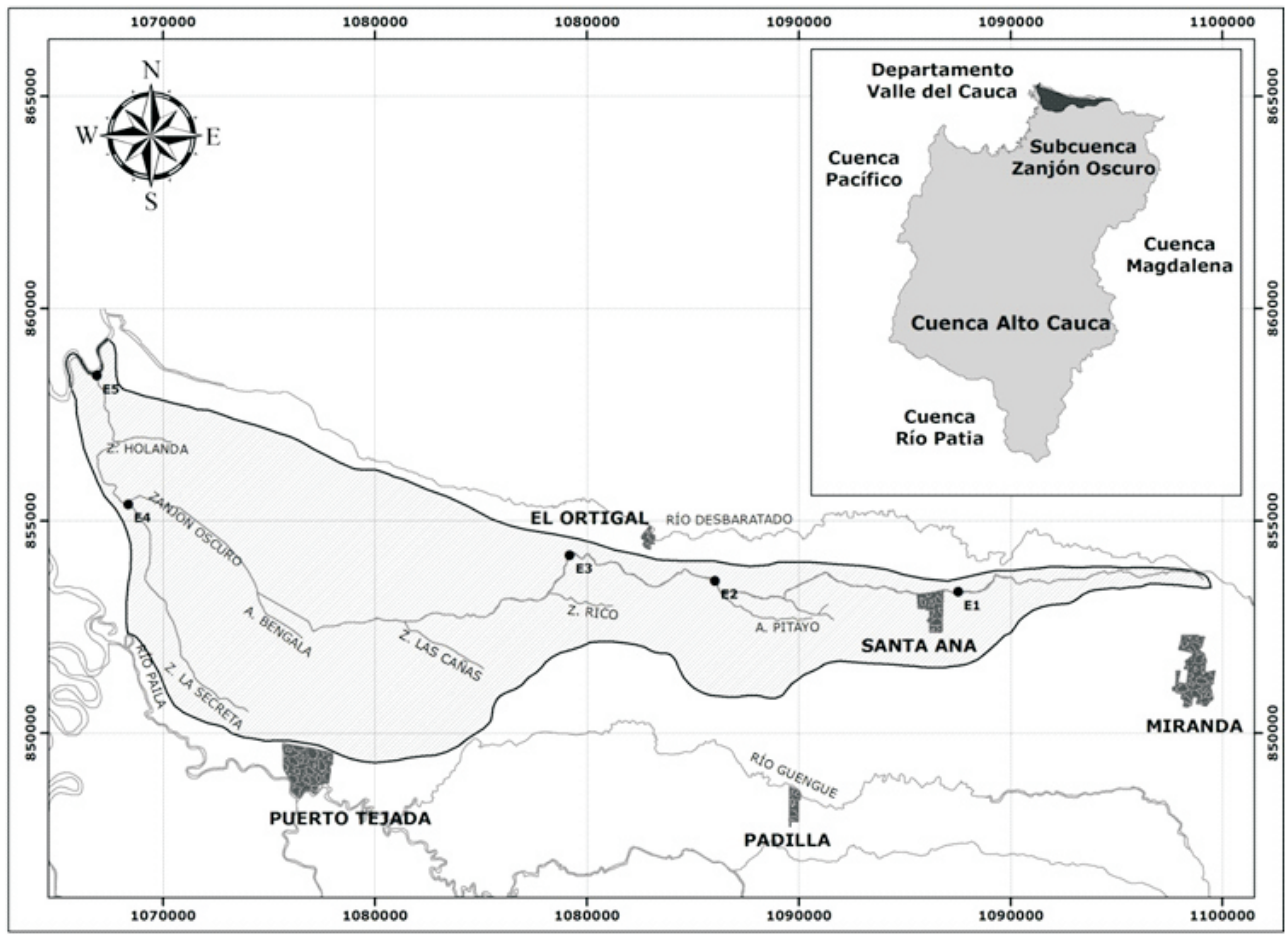

Figura 2. Localización Subcuenca Zanjón Oscuro 


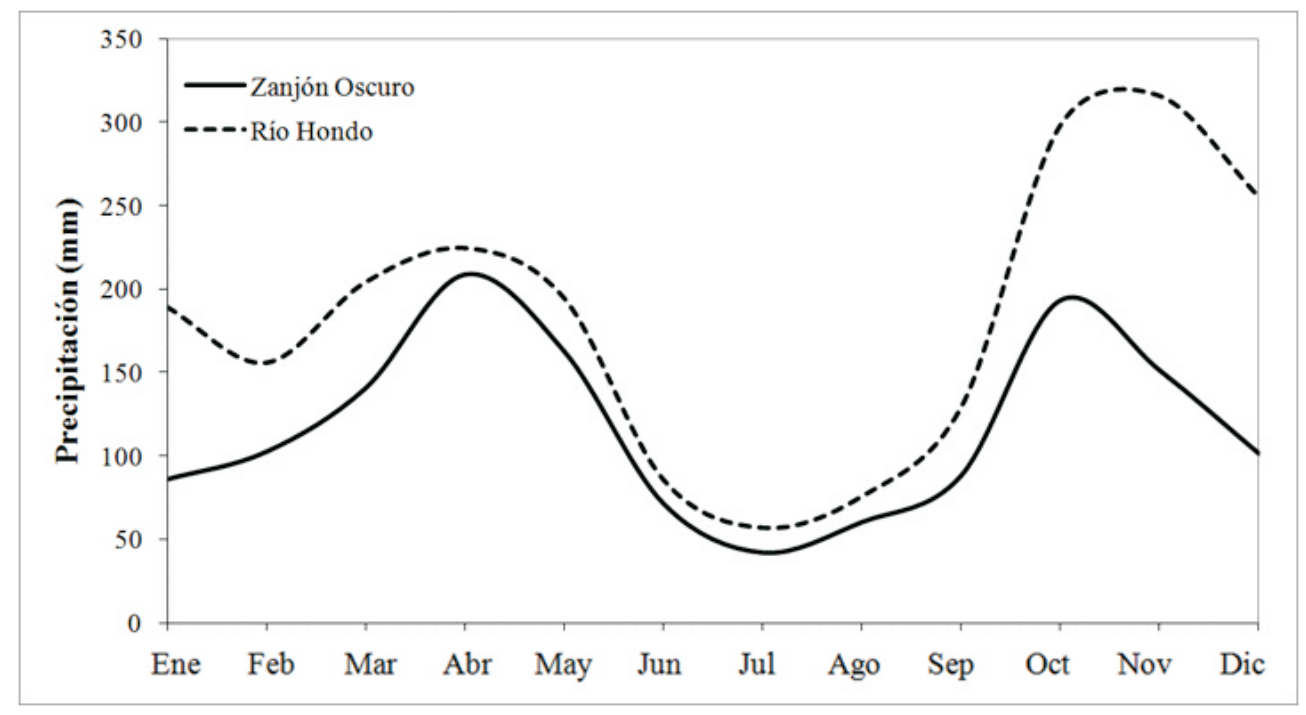

Figura 3. Precipitación media

- En la Subcuenca Zanjón Oscuro se establecieron cinco estaciones de muestreo durante los meses de Marzo, Julio y Octubre, así: sobre la acequia Santa Ana, antes del vertimiento de aguas residuales domésticas (ARD) (E1) y después de la planta de tratamiento de aguas industriales (PTAR) del Ingenio del Cauca (E2); sobre Zanjón Oscuro después del corregimiento El Ortigal (E3), antes del Zanjón La Secreta que drena las aguas residuales de Puerto Tejada (E4) y en la desembocadura al río Cauca donde lleva alta carga contaminante(E5).

- En la subcuenca Río Hondo con tres estaciones de muestreo durante los meses de Septiembre y Noviembre, así: sobre el Río Hondo en el sector Los Dos Brazos donde se realizan actividades de extracción de material de arrastre y recreación los fines de semana (E1), antes de la desembocadura del río Robles que recibe aguas grises de minifundios (E2) y después de la desembocadura del río las Piedras donde a esta altura ha recibido las aguas grises del sector urbano rural de la ciudad de Popayán y las aguas del río Robles (E3).

La selección de los tres puntos de monitoreo en cada subcuenca (Figuras 1 y 2), se realizaron teniendo en cuenta la zona alta, media y baja; además del acceso al lugar, los vertimientos de mayor impacto y la población asentada.

\subsection{Revisión de información secundaria}

Se revisaron los registros hidroclimatológicos y fisicoquímicos en las subcuencas objeto de estudio. Con esta información se definió el periodo de lluvias, paso seguido, se construye una base de datos con registros de 30 años, desde 1970 a 2000, y se establecen los puntos de muestreo en la zona de estudio.

\subsection{Trabajo de campo}

En cada punto de monitoreo se tomaron los siguientes parámetros in situ: $\mathrm{pH}$, conductividad, oxígeno disuelto y caudal. En el Laboratorio Ambiental de la Corporación Autónoma del Cauca (CRC) se determinó la demanda bioquímica de oxígeno $\left(\mathrm{DBO}_{5}\right)$, sólidos disueltos totales (SDT), Nitratos $\left(\mathrm{NO}_{3}^{-}\right)$, Fosfatos $\left(\mathrm{PO}_{4}{ }^{-}\right)$, coliformes fecales y totales.

\subsection{Determinación de la calidad}

Dado que uno de los principales usos del agua en río Hondo es para consumo y la de Zanjon Oscuro es de riego, se determinó que los indicadores más adecuados son el ICA-NSF y el ICOMO, los cuales incluyen las características fisicoquímicas y microbiológicas determinadas: 


\subsubsection{Indicador de contaminación del agua por materia orgánica "ICOMO"}

Este indicador fue desarrollado por Ramírez, et al. (1998), a partir de estudios fisicoquímicos, microbiológicos y limnológicos realizados en la industria petrolera para condiciones de ríos de Colombia. Utiliza las variables de $\mathrm{DBO}_{5}$, coliformes totales y porcentaje de saturación de oxígeno, donde las dos primeras reflejan fuentes diversas de contaminación orgánica y la tercera expresa la respuesta ambiental del cuerpo a este tipo de polución. La fórmula está dada por la Ec. (2):

$$
\text { ICOMO }=\frac{1}{3}\left(I_{\mathrm{DBO}_{5}}=I_{\text {Coliformes }}=I_{\text {Oxigeno\% } \%}\right)
$$

Donde: $I_{D B O_{5}}==0.05=0.70 \log _{10} D B O_{5}(\mathrm{mg} / \mathrm{L})$ Si: $\mathrm{DBO}_{5}>30(\mathrm{mg} / \mathrm{L})=1, \mathrm{DBO}_{5}<2(\mathrm{mg} / \mathrm{L})=0$.

$I_{\text {Coli.Tot. }}==1.44=0.56 \log _{10}$ Col.Tot.$(N M P / 0.1 L)$, Si: Col. Tot. $>20000(\mathrm{NMP} / 0,1 \mathrm{~L})=1$, Col. Tot. $<500(\mathrm{NMP} / 0.1 \mathrm{~L})=0 I_{\text {Oxizeno } \%}=1=0.01$ Oxígeno $\%$, Si: Oxígeno $(\%)>100 \%=0$

El valor de este indicador oscila de 0 a 1 y se clasifica en cinco categorías de contaminación: Ninguna (0 - 0.2), Baja ( $>0.2-0.4)$, Media $(>0.4$ $0.6)$, Alta $(>0.6-0.8)$ y Muy Alta $(>0.81)$.

\subsubsection{Indicador de calidad de agua, ICA-NSF}

Fue desarrollado por "The National Sanitation Foundation" de los Estados Unidos para la evaluación de la calidad fuentes para consumo. Utiliza nueve parámetros en su determinación que son: cambio de temperatura, $\mathrm{pH}, \mathrm{DBO}_{5}, \mathrm{OD}$, coliformes fecales, $\mathrm{NO}_{3}-\mathrm{N}$, fosfatos totales; turbiedad y sólidos disueltos totales (SDT) (NFS, 2006). Debido a que el cambio de temperatura no se determino el ICA-NSF se modificó utilizando ocho de los nueve parámetros, la formula general utilizada se presenta en la Ec. (3).

$$
I C A=N S F_{\text {Modificado }}=\underset{i=1}{=}\left(S u b_{\mathrm{i}}^{*} w_{\mathrm{i}}\right)
$$

Donde:

$\mathrm{Sub}_{\mathrm{i}} \quad$ Subíndice del Parámetro i

Wi Factor de Ponderación para el Subíndice i, así: OD 0.18, Coliformes fecales 0.16 ; $\mathrm{pH} 0.13$;
$\mathrm{DBO}_{5}$ 0,11; $\mathrm{NO}_{3}-\mathrm{N} \mathrm{0,11,} \mathrm{Fosfatos} \mathrm{totales} \mathrm{0,11;}$ Turbiedad 0,09 y SDT 0,09.

El valor de este indicador oscila entre 0 a 100 y se clasifica en cinco categorías de calidad: Muy Mala (0 - 25), Mala (26 - 50), Media (51 - 70), Buena (71 -90) y Muy Alta (91 100).

\subsection{Determinación de la oferta hídrica neta}

La oferta hídrica de una cuenca, es el volumen disponible para satisfacer la demanda generada por las actividades sociales y económicas del hombre, para la determinación en las subcuencas en estudio se realiza de acuerdo con la información disponible y sus características físicas, lo cual permite seleccionar la metodología de Relación lluvia escorrentía; aplicable en cuencas cuyas áreas de drenaje sean inferiores a $250 \mathrm{~km}^{2}$, cuencas poco instrumentadas $\mathrm{y}$ en consecuencia no cuentan con registros de caudal para la estimación de la oferta superficial mensual (MAVDT, 2004).

El conocimiento del caudal del río, su confiabilidad y extensión de la serie de registros históricos son variables y pueden influir en la estimación de la oferta hídrica superficial, en este caso mediante la Relación lluvia escorrentía (USACE, 2000), donde se utilizó toda la información disponible de las estaciones hidrométricas presentes en la zona de estudio y los aforos realizados en campo (CRC-UNIVALLE, 2007).

La oferta hídrica neta resulta de aplicar la reducción por caudal ecológico y calidad del agua a la oferta hídrica total (Ec. (1)). Para la reducción por caudal ecológico se tomó el $25 \%$ del caudal medio mensual multianual más bajo de la corriente en estudio. En el caso de la reducción por calidad del agua se propone el siguiente procedimiento:

Para evaluar la reducción por el contenido de materia orgánica y su uso doméstico, se determinaron los indicadores ICA-NSF e ICOMO, los cuales se obtienen a partir de la combinación de parámetros fisicoquímicos y microbiológicos en cada una de los cauces. A cada uno de estos se les asignó una reducción que oscila entre 0 y $100 \%$, como lo muestra la Ec. (4): 


$$
\begin{gathered}
\% R_{\mathrm{ICA}-\mathrm{NSF}}=100=I C A_{\mathrm{NSF}} \\
\% R_{\mathrm{ICO}}=I C O * 100
\end{gathered}
$$

Los porcentajes obtenidos se relacionan directamente con el aforo realizado el día del monitoreo en cada una de las estaciones. Lo ideal es realizar el cálculo por mes.

Para determinar la reducción total de la subcuenca se sumó cada uno de las reducciones aplicadas en cada sector (punto de muestreo); el total representa la cantidad a reducir para toda la subcuenca por mes, este valor se expresa en $\mathrm{m}^{3} / \mathrm{mes}$.

Debido a que no se tienen datos de calidad para cada mes, ni los respectivos aforos en cada punto de monitoreo; para encontrar la reducción para cada mes se seleccionó el caudal medio mensual y se le aplicó el porcentaje de reducción más alto encontrado a lo largo de la subcuenca, que corresponde a la estación de mayor contaminación.

Con el fin de comparar estos resultados con la normatividad se determinan la oferta hídrica neta aplicando la Resolución 0865 del 2004, donde las reducciones por caudal ambiental y calidad del agua se determinan a partir del $25 \%$ del caudal medio mensual multianual más bajo.

Si al aplicar las reducciones por calidad y caudal ecológico para determinar la oferta hídrica neta, se genera un valor negativo significa que no hay disponibilidad del recurso para el uso que se definió, en este caso uso doméstico y agrícola, por tanto se deben encender las alarmas locales y proceder a definir acciones para la recuperación hídrica de la subcuenca.

\section{Resultados y Discusión}

\subsection{Evaluación de la calidad}

En la Figura 4 se presentan los resultados para los indicadores de calidad y de contaminación en cada una de las estaciones, indicando los valores máximos, mínimos y la media, encontrando: que en la subcuenca Zanjón Oscuro después de la segunda estación la calidad y contaminación se clasifica entre mala (ICA-NSF: 30 a 50) y muy alta (ICOMO: 0.6 a 1.0) respectivamente; este impacto es provocado por el vertimiento de aguas residuales domésticas e industriales sobre este cauce después de la primera estación.
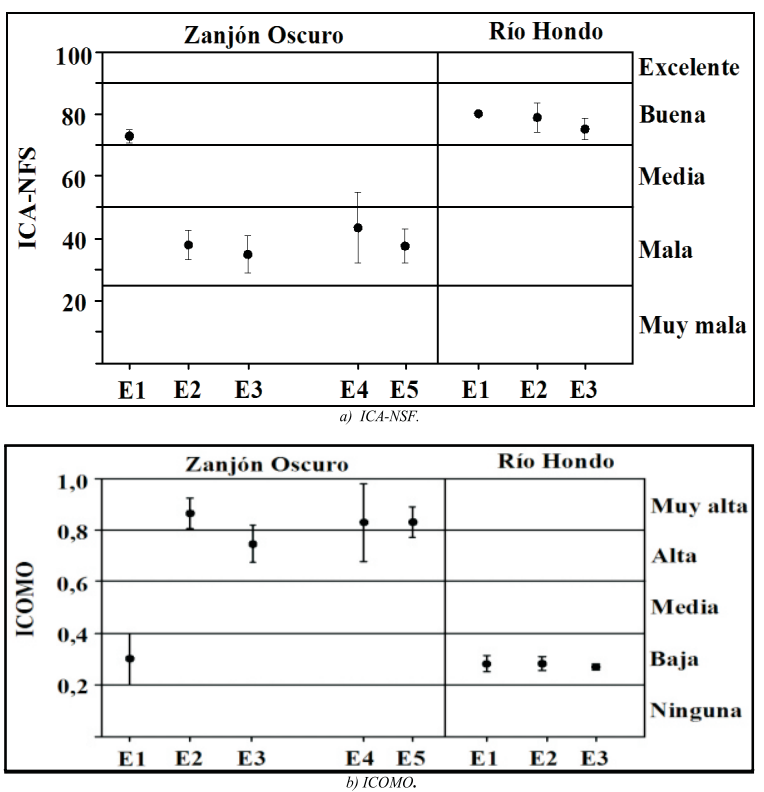

Figura 4. Tendencia del ICA-NFS e ICOMO en la zona de estudio

En el caso de la subcuenca río Hondo se encontró que los niveles de calidad y contaminación se mantienen en rangos de buenos (ICA-NSF 70 a 90) y bajos (ICOMO 0.2 a 0.4 ) respectivamente; encontrando que el efecto producido por aguas residuales domésticas provenientes de las veredas localizadas en los municipios de Popayán y El Tambo es bajo, por ser un río de montaña, lo cual favorece la oxigenación y además los tiempos de retención son bajos (6 horas en $60 \mathrm{~km}$ ), en comparación con Zanjón Oscuro que está ubicada en una zona plana donde los tiempos de retención son superiores ( 8 horas en $34 \mathrm{~km}$ ). En general se encontró que el principal agente de contaminación se debe a la materia orgánica, proveniente en gran porcentaje de aguas residuales domésticas.

\subsection{Oferta hídrica neta}

Teniendo en cuenta los resultados de calidad del agua se determinó los factores de reducción por calidad, para cada mes del año, como se presenta en la tabla 1. Como se observa los porcentajes de reducción estimados a partir de los indicadores 
ICA-NSF e ICOMO en la Subcuenca Zanjón Oscuro oscilan entre 57 y $91 \%$, valores superiores a los encontrados en río Hondo. Cuando se usa el indicador ICOMO los valores de reducción son superiores, debido a que el tipo de contaminación predominante es por materia orgánica.

De lo anterior se establece una relación directa entre los porcentajes de reducción, la calidad del recurso hídrico y la temporalidad, valores que afectan la oferta hídrica total (OHT). En la Figura 5 se comparan la cantidad en $\mathrm{m}^{3} /$ mes estimada a partir de los indicadores ICA-NSF e ICOMO y la resolución 0865 del 2004 en la zona de estudio.
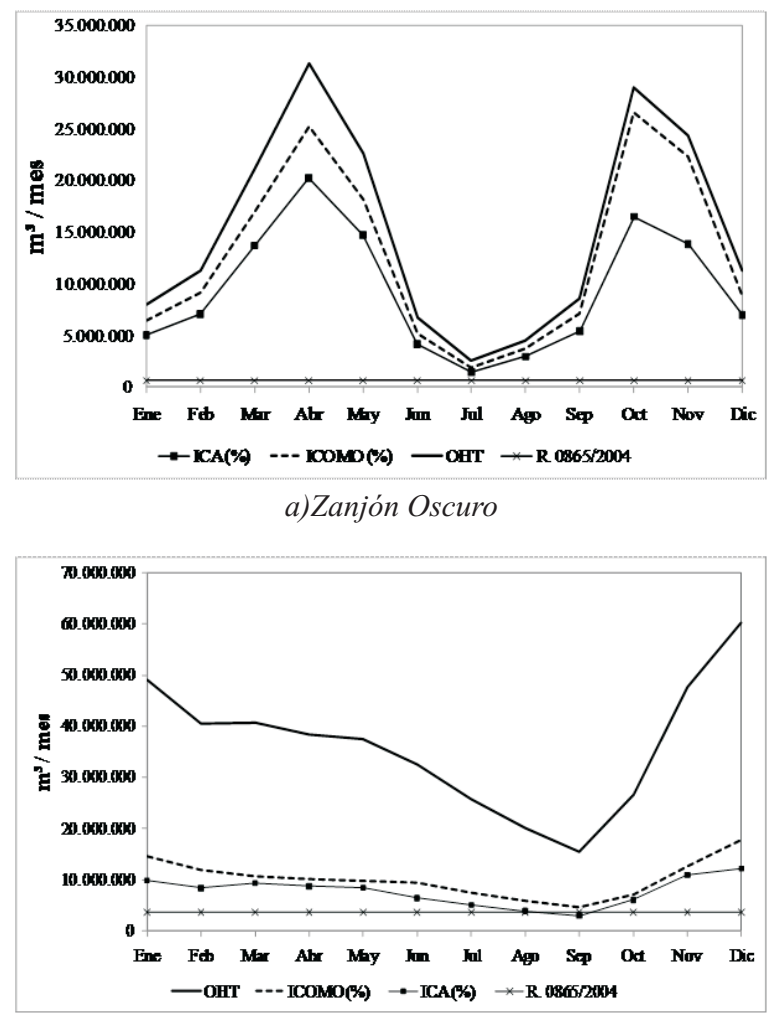

b) Río Hondo

Figura 5. Reducción por calidad del agua en la Zona de estudio

Encontrando que la reducción de calidad a partir de la metodología propuesta se ajusta a la variabilidad temporal en comparación con la resolución, que mantiene un valor constante durante todo el año. Es importante observar que los caudales de reducción son mayores para el caso de la Subcuenca de Zanjón Oscuro donde la contaminación es alta, mientras que en río Hondo es menor y llega a niveles muy bajos en el mes de septiembre. En la Figura 6, se compara la oferta hídrica neta $(\mathrm{OHN})$ estimada a partir de la reducción por calidad y caudal ecológico con la oferta hídrica total (OHT):
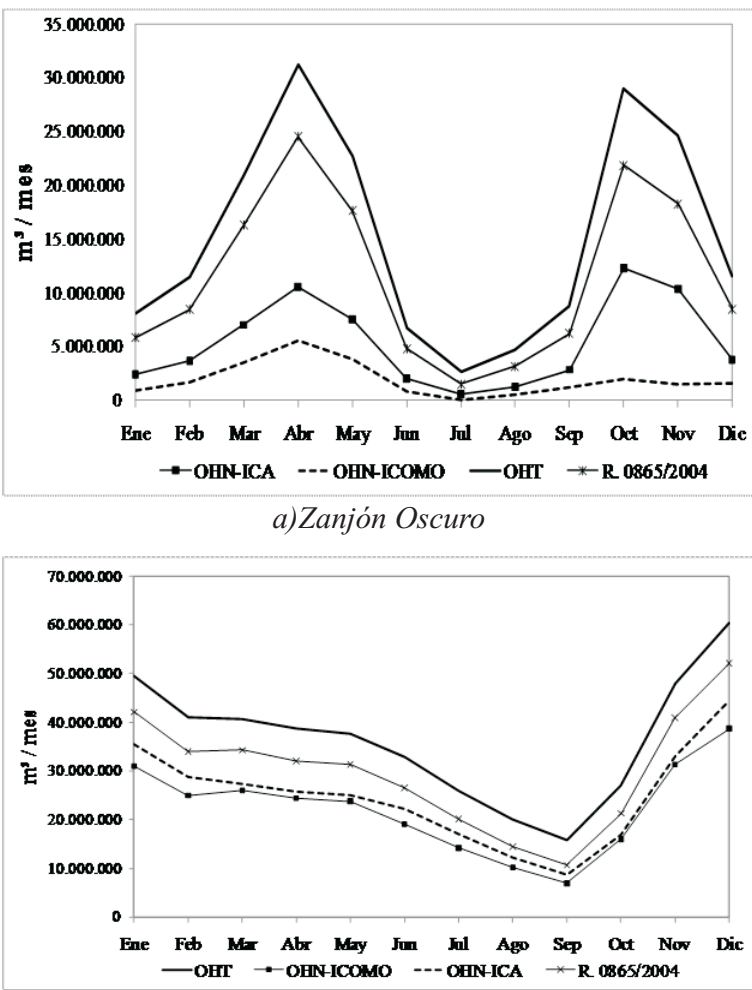

b) Rio Hondo

Figura 6.Comparación oferta hídrica neta estimada con la oferta hídrica total en la zona de estudio

En las dos subcuencas la OHN calculada a partir de los indicadores son menores en comparación a la resolución, además en Zanjón Oscuro toma valores cercanos a cero entre los meses de Junio y Agosto, indicando que la disponibilidad del recurso debe restringirse, debido al alto grado de contaminación por materia orgánica.

Al aplicar esta metodología se encontró una forma práctica y sencilla de establecer una relación directa entre las condiciones de la calidad del recurso hídrico y la reducción para su disponibilidad. 


\section{Conclusiones}

- Los resultados mostraron que la subcuenca Zanjón Oscuro presentó niveles de contaminación muy altos en el 2006 (ICOMO 0.6 a 1.0), por lo que la reducción del $25 \%$ por calidad que sugiere la resolución 0865 no refleja el estado de este cauce. Por lo tanto es importante aplicar factores más estrictos, como el propuesto por la metodología, que permitan estimar la oferta hídrica neta a partir de una relación directa con los estudios de calidad del agua.

- La metodología planteada para la determinación de la reducción por calidad del agua es sencilla y fácil de implementar. Donde se consigue establecer que para fuentes altamente contaminadas se deben dar restricciones de uso doméstico, casi en un $100 \%$.

- El procedimiento empleado puede mejorarse usando indicadores de calidad del agua de tipo mixto, donde se integren características fisicoquímicas y biológicas de la fuente.

- El indicador que se utiliza para la evaluación del recurso hídrico debe ser seleccionado de acuerdo al uso de la fuente, por ejemplo: si esta es para consumo usar ICA-NSF y para otros usos ICOMO, éste último porque determina la cantidad de materia orgánica, la cual limita varios usos como la recreación, doméstico y agricultura.

- El monitoreo debe ser mensual o trimestral, esto depende del tipo de uso de la fuente y el impacto en la calidad del agua, además la aplicación de esta metodología debe incluir la toma del caudal en la estación.

- La reducción por caudal ecológico se calculó al aplicar el $25 \%$ al caudal medio mensual multianual más bajo de la corriente en estudio, como lo recomienda la Resolución 0865 de 2004; aunque ésta no es la metodología más adecuada de acuerdo con la información disponible, se ajusta a los datos que se tienen. Para una mayor precisión se deben adelantar investigaciones al respecto en busca de una metodología integral para la determinación del régimen del caudal ambiental, que tenga en cuenta no solo variables hidrológicas como lo plantea la resolución 0865 de 2004, si no que tome en cuenta variables de tipo hidrológico, hidráulico y simulación de hábitat.

Esta investigación hace parte del proyecto "Desarrollo de una metodología para la determinación del índice de escasez y cálculo de la tasa por uso del agua, en trece (13) subcuencas hidrográficas del Departamento del Cauca" desarrollado por el grupo de investigación en Ingeniería de los Recursos Hídricos y Desarrollo de Suelos (IREHISA) de la Escuela de Ingeniería de los Recursos Naturales y del Ambiente (EIDENAR) de la Universidad del Valle, el cual permitió a la Corporación Autónoma del Cauca (CRC) tener herramientas para la gestión del recurso hídrico.

\section{Agradecimientos}

Agradecemos al grupo IREHISA de la Universidad el Valle por el apoyo técnico y científico para la realización de este artículo. Y a la $\mathrm{CRC}$, por el apoyo económico y administrativo para el desarrollo del proyecto.

\section{Referencias bibliográficas}

Castro, L., Carvajal, Y., \& Monsalve, E. (2006). Enfoques Teóricos para definir el caudal ambiental. Revista Ingeniería y Universidad, 10 (2), 74-101.

Castro, L., \& Carvajal, Y. (2009). Metodologías para determinar el caudal ambiental. En: J. Cantera (editor), El caudal ambiental: conceptos, experiencias y desafios. Universidad del Valle. (Capitulo 5).

CRC (Corporación Autónoma Regional del Cauca). (2001). Evaluación de la demanda / oferta de agua de la subcuenca del río La Quebrada, zona norte del departamento del Cauca. Documento preparado para la Oficina de Gestión Ambiental, Popayán, Colombia.

CRC (Corporación Autónoma Regional del Cauca) \& FONAN (Fondo Nacional Ambiental) (2000). Monitoreo ambiental con énfasis en el recurso hídrico Cuenca Cauca, Departamento del Cauca. Fase I. Documento preparado para la Oficina de Investigaciones Ambientales, Popayán, Colombia. 
CRC \& UNIVALLE (Universidad del Valle). (2007). Desarrollo de una metodología para la determinación del índice de escasez y cálculo de la tasa por uso del agua, en trece (13) subcuencas hidrográficas del Departamento del Cauca. Documento preparado para la Oficina de Investigaciones Ambientales, Popayán, Colombia.

DANE (Departamento Administrativo Nacional de Estadística). (2005). Censo General 2005. Nivel Nacional. Bogotá, Colombia.

Fernández, N., \& Solano, F. (2005). Índices de calidady de contaminación del agua. Universidad de Pamplona. Colombia.

Gottschalk, L. \& Krasovskaya, I. (2010). Statistical river flow descriptors for environmentalflow assessment. 340, s 350-357.

Guhl, E. (2006). Peligros y soluciones a la escasez y contaminación creciente del agua. El Tiempo, 5 de abril de 2006. Bogotá, Colombia.

IDEAM (Instituto de Hidrología, Meteorología y Estudios Ambientales). (2005). Estudio Nacional del Agua. Documento preparado para: Ministerio de Medio Ambiente, Bogotá, Colombia.

IDEAM, 2010. Estudio Nacional del Agua 2010. Instituto de Hidrología, Meteorología y Estudios Ambientales. Bogotá D.C.

Magaña, V. (2004). El cambio climático global: Comprender el problema: EN Cambio climático: una mirada desde México, México. INE, 18.

Malvezzi, R. (2006). La cuestión del agua en América Latina. En: http://www.bolpress.com. Visitada: Agosto de 2009

Marín, R. (2003). Colombia Potencia Hídrica. Subdirección de Hidrología. Instituto de Hidrología, Meteorología y Estudios Ambientales (IDEAM). Bogotá, Colombia.

MAVDT (Ministerio de Ambiente, Vivienda y Desarrollo Territorial). (2004). Resolución 0865 de Julio 22 de 2004. Por la cual se adopta la metodología para el cálculo del índice de escasez para aguas superficiales. Bogotá, Colombia.
NSF (National Sanitation Fundation). (2006). Consumer Information: Water Quality Index (WQI).

Http://www.nsf.org/consumer/just_for_kids/wqi.asp Visitada: Enero de 2008

OEA (Organización de Estados Americanos). (2004). Metodología para el cálculo del índice de escasez de agua superficial. Documento preparado para el Sistema de Información del Medio Ambiente, SIMA, Instituto Lima, Perú.

Palau, I. (2003). Régimen ambiental de caudales: estado del arte. In proceedings: Régimen Ambiental de Caudales, del 29 de septiembre al 3 de octubre de 2003, Unidad Docente de Hidráulica e Hidrología, E.U.I.T., Forestal, Universidad Politécnica de Madrid, Cuenca, España, 1-8.

Ramírez, A., Restrepo, R., \& Viña, G. (1998). Cuatro índices de contaminación para caracterización de aguas continentales. Formulación y Aplicación. Ciencia, Tecnología y Futuro 1(3), 135-153.

Rivera, H., Dominguez E., Marin, R., \& Vanegas, R., (2004). Metodología para el cálculo del índice de escasez. Documento preparado para el Instituto de Hidrología, Meteorología y Estudios Ambientales IDEAM. Sistema Nacional Ambiental. Bogotá, Colombia.

Samboní, N. (2009). Estimación del índice de escasez empleando los indicadores de calidad y contaminación del agua en la cuenca alta del Río Cauca. Tesis de Maestría. Escuela de Ingeniería de Recursos Naturales y del Ambiente. Facultad de Ingeniería, Universidad del Valle, Cali, Colombia.

Samboni, N., Carvajal, Y., \& Escobar, L. (2007). Parámetros fisicoquímicos como indicadores de calidad y contaminación del agua, estado del arte. Ingeniería e Investigación 27 (3), 172-181.

Sommer, M. (2000). Agua, no al desperdicio, no a la escasez. En: http:// www.waste.ideas.es Visitada: Septiembre de 2009 
USACE (United States Armyo Corps of Engineers). (2000). Hydrologic Modeling System HEC-HMS Technical Reference Manual, Hydrologic Engineering Center.

Yepe, M. (2008). El derecho humano al agua. http://www.ecoportal.net/content/view/full/ 75376 